\title{
32-Gbps single silicon microring resonator-loaded Mach-Zehnder modulator
}

\author{
Yuki Yabushita, Hiroki Takazawa, Yasuo Kokubun, and Taro Arakawa* \\ Graduate School of Engineering, Yokohama National University, Yokohama, Kanagawa 240-8501, Japan \\ *E-mail: arakawa-taro-vj@ynu.ac.jp \\ Received January 31, 2018; accepted May 12, 2018; published online July 18, 2018
}

Design and high-speed modulation of a compact and low-voltage silicon microring resonator-loaded Mach-Zehnder modulator (Si MRR-MZM) with a lateral P-N junction are discussed. The driving voltage or the size of a Si MZM is expected to be significantly reduced owing to an enhanced phase shift in an MRR. Modulation characteristics of the MRR-MZM are analyzed on the basis of different device parameters, such as the coupling efficiency $K$ and round-trip length of an MRR. Si MRR-MZMs with different device parameters are fabricated using a complementary metal-oxidesemiconductor (CMOS)-compatible process and their high-speed modulation characteristics are compared. $\mathrm{A}$ half-wave voltage of $3.4 \mathrm{~V}$ is demonstrated in an MRR-MZM with $K=0.12$ and a round-trip length of $128 \mu \mathrm{m}$, and the product of the half-wave voltage and the length of a phase shifter $\left(V_{\pi} L\right)$ was decreased to $0.036 \mathrm{~V} \mathrm{~cm}$. The $3 \mathrm{~dB}$ bandwidth of an MRR-MZM with $K=0.21$ and a round-trip length of $180 \mu \mathrm{m}$ is measured to be approximately $16 \mathrm{GHz}$, and nonreturn-to-zero (NRZ) modulation up to $32 \mathrm{Gbps}$ is successfully demonstrated for an operation voltage of $4.0 \mathrm{~V}$. (C) 2018 The Japan Society of Applied Physics

\section{Introduction}

Silicon (Si) Mach-Zehnder modulators (MZMs) are essential devices in high-speed optical communication networks and for optical interconnection in silicon photonics circuits owing to their compactness and low-cost fabrication using a complementary metal-oxide-semiconductor (CMOS)-compatible process. MZMs have the advantages of not only lowchirp modulation characteristics but also suitability for spectrally efficient advanced modulation formats such as quadrature phase-shift keying (QPSK). ${ }^{1,2)}$ So far, high-speed Si MZMs have been developed for on-off keying (OOK), ${ }^{3-9)}$ QPSK, ${ }^{10-14)}$ and higher-efficiency modulation formats. ${ }^{15-17)}$ However, the footprint of Si MZMs is usually relatively large owing to their low phase modulation efficiency of the carrier plasma effect in a lateral or interleaved $\mathrm{P}-\mathrm{N}$ junction. A typical driving voltage and product of the half-wave voltage and the length of a phase shifter $V_{\pi} L$ of a normal Si MZM are approximately $5.0 \mathrm{~V}$ and $2.5 \mathrm{~V} \mathrm{~cm}$, respectively. In addition, compact slow-light MZMs with photonic crystals (PCs) have also been demonstrated. ${ }^{18,19)}$ Even though PC-based MZMs have a small footprint, they require a complicated and precise fabrication process for the PC structure.

On the other hand, microring resonators (MRRs) are also important devices for large-scale integrated optical crossconnect circuits using multiple-wavelength channels owing to their high functionality and compactness. ${ }^{20,21)}$ Using $\mathrm{Si}$ MRRs, high-order MRR filters and high-speed MRR optical modulators and switches have been demonstrated. ${ }^{22-27)}$ Moreover, a nonlinear phase-shift effect in an $\mathrm{MRR}^{28}$ ) has recently attracted interest, and Mach-Zehnder interferometers (MZIs) incorporating the nonlinear phase-shift effect have also been extensively studied owing to their compact size and low driving voltage. To date, compound-semiconductor- and Si-MRR-assisted MZMs (MRR-MZMs) and switches have been developed using the carrier plasma effect, ${ }^{29-35)}$ the thermo-optic (TO) effect, ${ }^{36,37)}$ and an electric-field induced change in the refractive index, ${ }^{38,39)}$ and many of them are driven by a pair or pairs of MRRs. Although a multiple MRR-MZM with push-pull driving is advantageous for symmetric switching and lowering driving voltage, the precise control of resonant wavelengths of two MRRs is required.

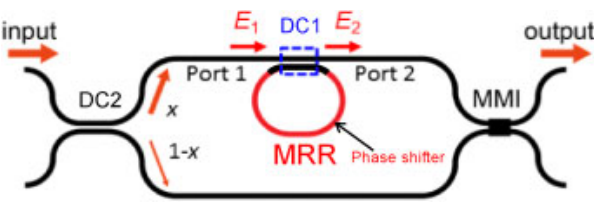

Fig. 1. (Color online) Schematic top view of Si MRR-MZM. Si ribwaveguide MRR is coupled with one of the arms of MZI through DC1. The red waveguide is a phase shifter. DC2 with an asymmetric split ratio is used to improve the extinction ratio.

In this paper, we discuss design and high-speed modulation of single Si MRR-MZMs ${ }^{32,40)}$ with different device parameters. The Si MRR-MZMs are fabricated using a CMOS-compatible process, and their high-speed modulation characteristics are compared. A half-wave voltage of $3.4 \mathrm{~V}$ is demonstrated in an MRR-MZM with $K=0.12$ and a roundtrip length of $128 \mu \mathrm{m}$. The $3 \mathrm{~dB}$ bandwidth of an MRR-MZM with $K=0.21$ and a round-trip length of $180 \mu \mathrm{m}$ is measured to be approximately $16 \mathrm{GHz}$, and nonreturn-to-zero (NRZ) modulation up to $32 \mathrm{Gbps}$ is successfully demonstrated for an operation voltage of $4.0 \mathrm{~V}$.

\section{Operation principle}

Figure 1 shows a schematic top view of the Si MRR-MZM. A $\mathrm{Si}$ rib-waveguide $\mathrm{MRR}$ with a lateral $\mathrm{P}-\mathrm{N}$ junction is coupled with one of the arm waveguides of an MZI through a directional coupler (DC) denoted as DC1, and it acts as a phase shifter through the carrier plasma effect in the depletion mode. An asymmetric DC (DC2) is used on the side of the input port, as discussed in Sect. 3.

Because of the enhanced phase shift in the MRR, ${ }^{28)}$ the phase of the light in the arm with the MRR is significantly changed by a small change in the refractive index of the MRR waveguide. If the light power losses at the DC are neglected, the effective phase $\phi_{\text {eff }}$, that is, the phase change of the optical electric field transmitted from Ports 1 to 2, and the transmittance $T$ of the light power from Ports 1 to 2 are given by $^{28)}$

$$
\phi_{\text {eff }}=\arg \left(\frac{E_{2}}{E_{1}}\right)
$$




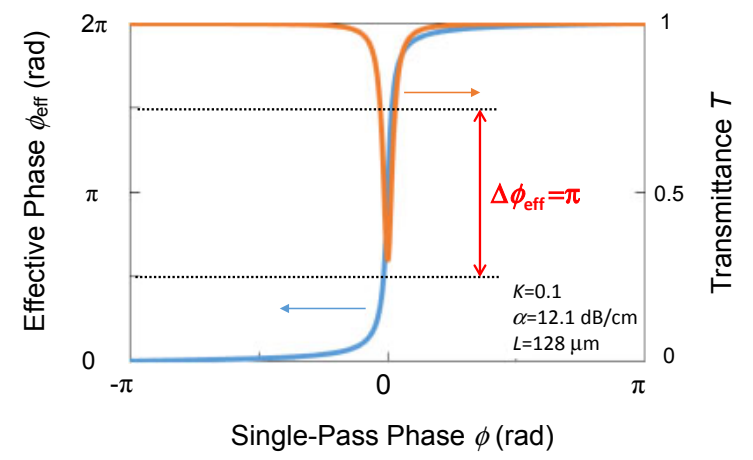

Fig. 2. (Color online) Calculated effective phase shift $\phi_{\text {eff }}$ and transmittance $T$ as functions of single-pass phase shift $\phi$ for $K=0.10$, $L=128 \mu \mathrm{m}$, and $\alpha=12.1 \mathrm{~dB} / \mathrm{cm}$.

$$
\begin{aligned}
&= \pi+\phi+\tan ^{-1}\left(\frac{r \sin \phi}{\eta a-r \cos \phi}\right) \\
&+\tan ^{-1}\left(\frac{\eta \operatorname{ar} \sin \phi}{1-\eta \operatorname{arcos} \phi}\right) . \\
& r= \sqrt{1-K}, \quad a=\exp \left(-\frac{\alpha L}{2}\right), \\
& T=\left|\frac{E_{2}}{E_{1}}\right|^{2}=\left|\frac{\eta^{2}\left(\eta^{2} a^{2}+r^{2}-2 \eta \operatorname{\eta rcos} \phi\right)}{\eta^{2} a^{2} r^{2}+1-2 \eta \cos \cos \phi}\right|,
\end{aligned}
$$

where $E_{1}$ and $E_{2}$ are respectively the optical electric fields at Ports 1 and 2. $\phi$ is the single-pass phase in the MRR, and $r$ and $K$ are respectively the coupling ratio of the optical electric field and the coupling efficiency of the light power at the DC. $\alpha$ and $L$ are the propagation loss coefficient in the MRR and the round-trip length of the MRR, respectively. $a$ and $\eta$ are the transmission coefficient of the electric field for round-trip propagation along the MRR and the coupling loss at the DC, respectively.

Figure 2 shows the effective phase shift $\phi_{\text {eff }}(\bmod 2 \pi \mathrm{rad})$ and the transmittance $T$ as functions of the single-pass phase shift $\phi(\bmod 2 \pi \mathrm{rad})$ for $K=0.10, L=128 \mu \mathrm{m}, \alpha=12.1$ $\mathrm{dB} / \mathrm{cm}$, and $\eta=0$. In the following theoretical discussion, these values of $\alpha$ and $\eta$ are used. In the vicinity of $\phi=0$, that is, in the on-resonance state, the marked nonlinearity and the single-pass phase shift are strongly enhanced. Here, we define the phase shift enhancement factor $F_{\text {pe }}$ as ${ }^{38,39)}$

$$
\begin{aligned}
F_{\mathrm{pe}} & =\frac{\Delta \phi_{\text {eff }}}{\Delta \phi} \\
& =\frac{3 \pi / 2-\pi / 2}{\left.\phi\right|_{\phi_{\text {eff }}=3 \pi / 2}-\left.\phi\right|_{\phi_{\text {eff }=\pi / 2}}} \\
& =\frac{\pi}{\left.\phi\right|_{\phi_{\text {eff }}=3 \pi / 2}-\left.\phi\right|_{\phi_{\text {eff }}=\pi / 2}} .
\end{aligned}
$$

I the case of $K=0.1$ and $L=128 \mu \mathrm{m}, F_{\mathrm{pe}}$ is approximately 31.5. Using this enhancement of the phase shift, the driving voltage of the MRR-MZM can be reduced to $1 / F_{\text {pe. The }}$ proposed devices are designed to modulate transverse electric (TE) mode lights and to be used in the $\mathrm{C}$ band.

\section{Device design and fabrication}

The Q factor and phase shift enhancement factor are the important indices for the characteristics of MRRs. The Q factor of the MRR is decided with the coupling efficiency $K$ at a DC between an MRR and a busline waveguide, and the

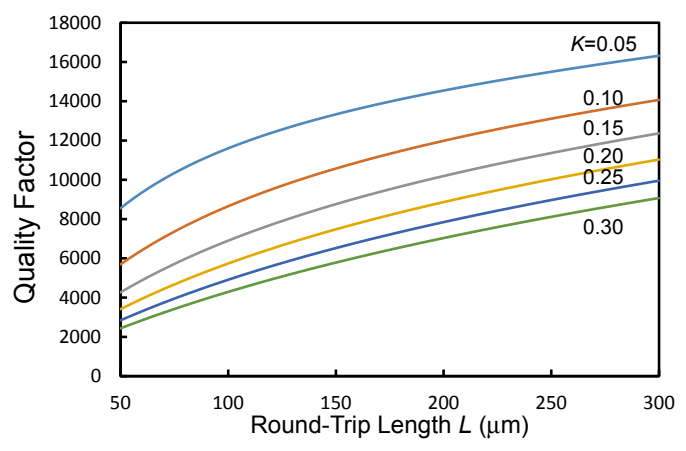

(a)

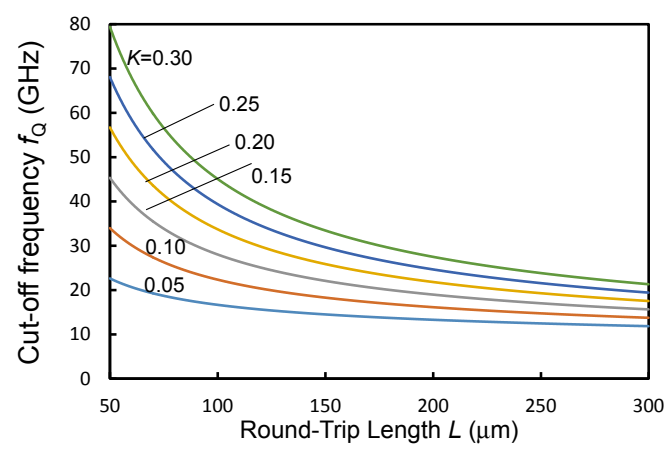

(b)

Fig. 3. (Color online) (a) Calculated relationship between the $\mathrm{Q}$ factor and round-trip length $L$ at various $K$ values. (b) Calculated cut-off frequency $f_{\mathrm{Q}}$ decided by the Q factor as a function of $L$ at various $K$ values.

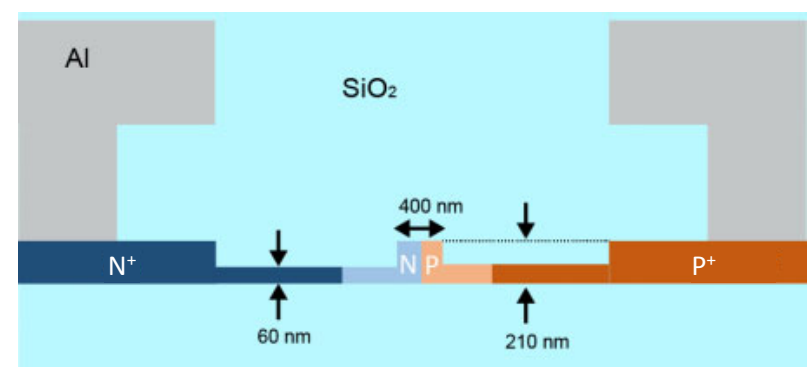

Fig. 4. (Color online) Schematic cross-sectional view of Si rib waveguide with lateral $\mathrm{P}-\mathrm{N}$ junction.

round-trip length $L$ of the MRR. Figure 3(a) shows the calculated relationship between the $\mathrm{Q}$ factor and round-trip length at various $K$ values. The $\mathrm{Q}$ factor increases with decreasing $K$ and increasing $L$, which leads to the larger phase enhancement effect. The modulation speed is limited by the $\mathrm{Q}$ factor and the $\mathrm{CR}$ time constant of the circuit. Figure 3(b) shows the calculated cut-off frequency $f_{\mathrm{Q}}$ decided by the $\mathrm{Q}$ factor as a function of $L$ at various $K$ values. To increase modulation speed limited by the $\mathrm{Q}$ factor, larger $K$ and smaller $L$ values are required.

The proposed MZM was fabricated on a silicon-oninsulator (SOI) substrate using a CMOS-compatible process. In the proposed MZM, a lateral $\mathrm{P}-\mathrm{N}$ junction is used in the MRR. Figure 4 shows a schematic cross-sectional view of the rib waveguide. The width and thickness of the rib waveguide are 0.40 and $0.21 \mu \mathrm{m}$, respectively. The thickness of the slabs connected to the $\mathrm{P}-\mathrm{N}$ junction is $0.06 \mu \mathrm{m}$.

To improve the extinction ratio of the MZM with singlearm driving, a DC with an asymmetric light power splitting 


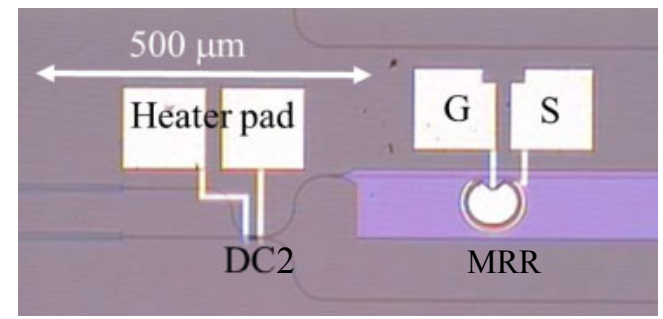

Fig. 5. (Color online) Optical microscopy image of the fabricated MRRMZM.

Table I. Designed device parameters.

\begin{tabular}{lcc}
\hline \multirow{2}{*}{ Device parameter } & \multicolumn{2}{c}{ Designed value } \\
\cline { 2 - 3 } & Device 1-1, 1-2 & Device 2 \\
\hline Round-trip length of MRR $L(\mu \mathrm{m})$ & 128 & 180 \\
Coupling efficiency $K$ & 0.10 & 0.15 \\
Quality factor & 10619 & 10376 \\
Branching ratio of DC $x$ & 0.58 & 0.57 \\
Phase shift enhancement factor $F_{\mathrm{pe}}$ & 31.5 & 20.1 \\
P/N doping concentration $\left(\mathrm{cm}^{-3}\right)$ & \multicolumn{2}{c}{$4.76 \times 10^{17}$} \\
\hline
\end{tabular}

ratio of $x: 1-x$ should be used instead of MMI couplers $(x=0.5)$ to reduce the imbalance between the light powers in the two arms. The splitting ratio for the maximum extinction ratio $x_{\mathrm{opt}}$ is given by

$$
x_{\mathrm{opt}}=\frac{T_{2}^{2}}{T_{1}^{2}+T_{2}^{2}},
$$

where $T_{1}$ and $T_{2}$ are the transmittances in the arms with and without the MRR, respectively. In the case of $K=0.1$, $L=128 \mu \mathrm{m}$, and $\alpha=12.1 \mathrm{~dB} / \mathrm{cm}$, the optimized $x_{\mathrm{opt}}$ is 0.58 .

The MRR-loaded MZMs were fabricated by a CMOScompatible process. Figure 5 shows an optical microscopy image of the fabricated device with an MRR with the roundtrip length of $180 \mu \mathrm{m}$. The total length of the device including the spot-size converters at the input and output ports is approximately $2 \mathrm{~mm}$. The designed device parameters for the MRR-MZMs are summarized in Table I. The doping concentrations of the $\mathrm{P}$ - and $\mathrm{N}$-doped regions were chosen with the constraints in the fabrication process considered. The gaps between the waveguides at DC1 and DC2 are both $0.45 \mu \mathrm{m}$. The coupling efficiency at DC1 and the splitting ratio at DC2 are controlled by changing the coupling length of the DC. The required coupling lengths for $K=0.1$ at DC1 and for $x=0.58$ at DC2 and are 4.5 and $9.8 \mu \mathrm{m}$, respectively. Even though a TiN heater is attached to DC2 for the fine adjustment of the splitting ratio, it was not used in the following experiments because it did not work effectively.

The phase shifters in the fabricated devices are 105 and $157 \mu \mathrm{m}$ for the devices with the round-trip lengths of 128 and $180 \mu \mathrm{m}$, respectively, because the DCs and their vicinities are non-phase-shifting waveguides.

\section{Modulation characteristics}

\subsection{Static modulation characteristics}

Figure 6 shows the measured spectral responses at the output port under various applied reverse voltages for Devices 1-1, $1-2$, and 2. The temperature of the modulators were kept at $15^{\circ} \mathrm{C}$ using a Peltier element device. In these cases, with the

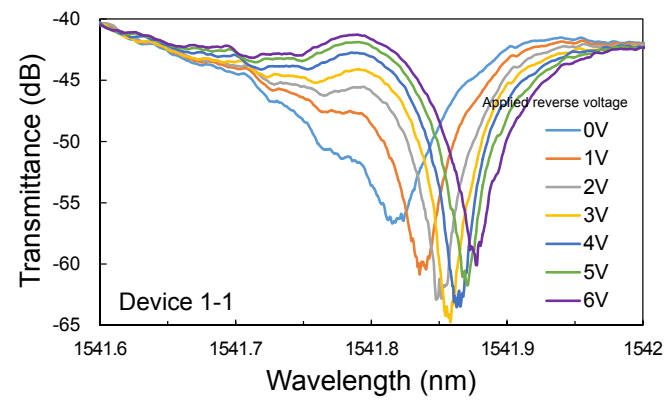

(a)

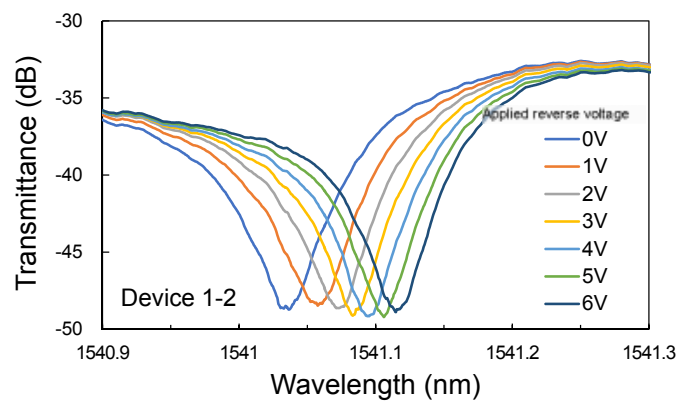

(b)

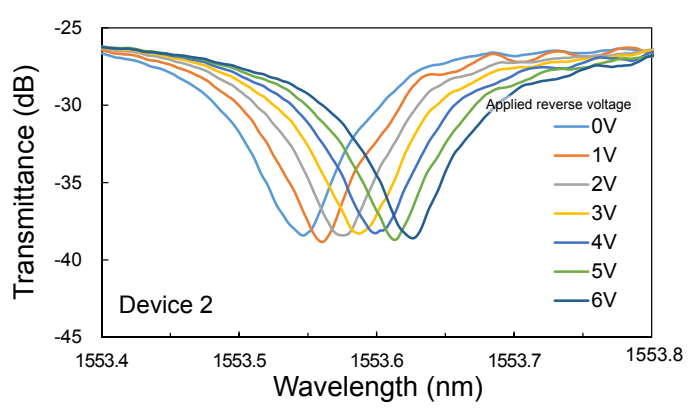

(c)

Fig. 6. (Color online) Measured spectral responses of the fabricated MRR-MZMs (Devices 1-1, 1-2, and 2) at output port under various applied reverse voltages.

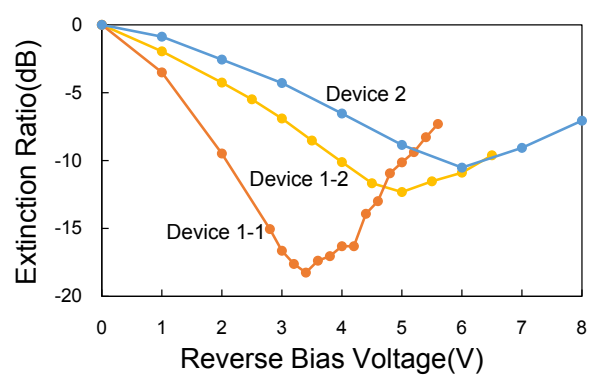

Fig. 7. (Color online) Measured extinction ratios of fabricated MRRMZMs (Devices 1-1, 1-2, and 2).

increase in applied reverse voltage, the shift of resonant dips to the longer wavelength side. The slope on the dip is slightly steeper for Device 1-1 than for Devices 1-2 and 2 owing to the larger phase shift enhancement. This steep slope leads to a low driving voltage.

Figure 7 shows the measured extinction ratios of Devices $1-1,1-2$, and 2 at wavelengths of $1541.86,1541.11$, and $1553.63 \mathrm{~nm}$, and the driving voltages of these devices are 3.4, 5.0 , and $6.0 \mathrm{~V}$, respectively. Owing to the enhanced phase shift in the MRR, the products of the half-wave voltage and phase shifter length $\left(V_{\pi} L\right)$ of as low as approximately 0.036 , 


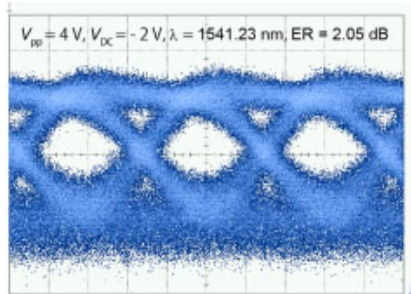

(a)

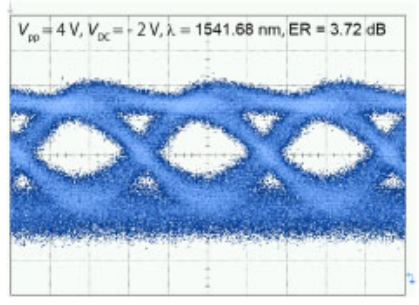

(c)

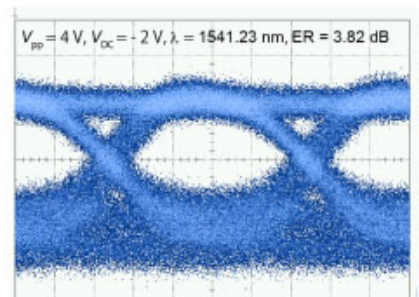

(b)

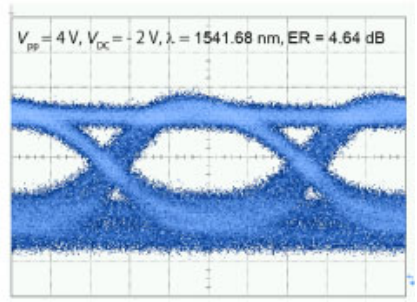

(d)
Fig. 8. (Color online) Measured eye patterns of fabricated MRR-MZM at $V_{\mathrm{pp}}=4.0 \mathrm{~V}$ (a) Device 1-2, $32 \mathrm{Gbps}$, (b) Device 1-2, $20 \mathrm{Gbps}$, (c) Device 2, $32 \mathrm{Gbps}$, and (d) Device 2, $20 \mathrm{Gbps}$ at wavelength of about $1541 \mathrm{~nm}$.

0.052, and $0.094 \mathrm{Vcm}$ for Devices 1-1, 1-2, and 2, respectively, were successfully achieved by single-arm driving using the MRR in the static modulation, with regard to the round-trip length of the MRR as the phase shifter length. The extinction ratios of Devices 1-1, 1-2, and 2 were $18.3,12.3$, and $10.5 \mathrm{~dB}$, respectively. The reason for the small extinction ratios of Devices $1-2$ and 2 is probably because of the fact that the coupling efficiencies in these devices are larger than the designed ones, as mentioned later, which deteriorated the balance between light powers and phases in both arms. The phase-shift enhancement factors for Devices 1-1, 1-2, and 2 were evaluated to be approximately 27, 22, and 14, respectively. The Q-factors of Devices 1-1, $1-2$, and 2 that are evaluated from each measured $F_{\mathrm{pe}}$ are approximately 9680,9260 , and 8630 , which correspond to the coupling efficiencies of $0.12,0.13$, and 0.21 , respectively. The discrepancies between the designed and measured coupling efficiencies are probably due to fabrication error.

\subsection{Dynamic modulation characteristics}

The characteristics of the high-speed modulation of the MRRMZMs were measured. The temperature of the modulators were kept at $15^{\circ} \mathrm{C}$ using a Peltier element device. Unfortunately, since we were unable to measure Device 1-1 owing to the large coupling loss with optical fibers, the modulation characteristics of Devices 1-2 and 2 were measured. Figure 8 shows the measured eye patterns of the optical modulation signals of the fabricated MRR-MZM at bit rates of 20 and $32 \mathrm{Gbps}$ using a bias voltage $V_{\text {bias }}$ of $2.0 \mathrm{~V}$ and a voltage swing $V_{\mathrm{pp}}$ of $4.0 \mathrm{~V}$ without pre-emphasis. The MZM was driven by a $2^{31}-1$-bit NRZ pseudorandom bit sequence (PRBS) signal. Clear eye openings were observed at 20 and $32 \mathrm{Gbps}$. The extinction ratios at $32 \mathrm{Gbps}$ were 3.7 and $2.0 \mathrm{~dB}$ for Devices 1-2 and 2, respectively. Figure 9 shows the measured eye patterns at bit rates of $32 \mathrm{Gbps}$ and $V_{\mathrm{pp}}$ of $2.0 \mathrm{~V}$. Even in these cases, the extinction ratios of 1.9 and $2.4 \mathrm{~dB}$ for Devices $1-2$ and 2 , respectively, were obtained.

Figure 10 shows the frequency characteristics of the transmitted light $\left(S_{21}\right)$ of the MRR-MZMs. The $3 \mathrm{~dB}$ cut-off frequencies of Devices $1-2$ and 2 were 12 and $16 \mathrm{GHz}$,

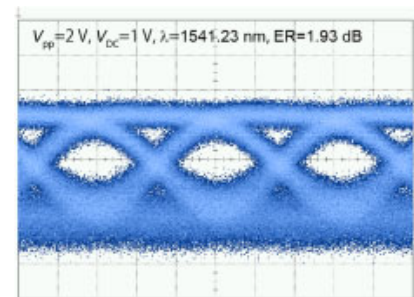

(a)

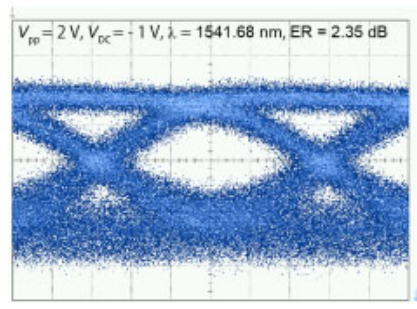

(b)
Fig. 9. (Color online) Measured eye patterns of fabricated MRR-MZM at 32 Gbps, $V_{\mathrm{pp}}=2.0$ V. (a) Device 1-2, (b) Device 2.

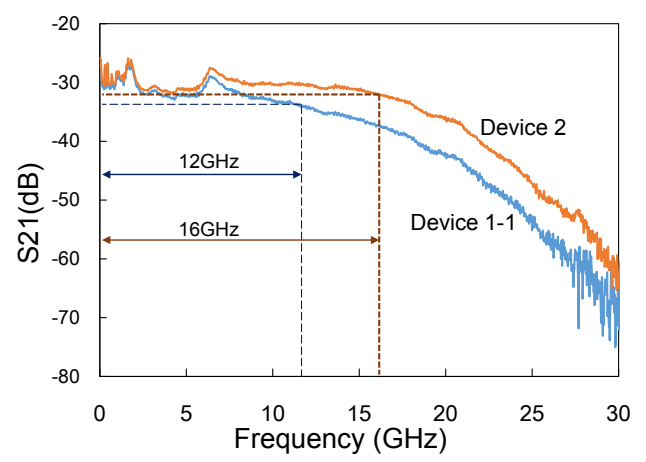

Fig. 10. (Color online) Frequency characteristics of transmitted light $\left(S_{21}\right)$ of the MRR-MZMs.

respectively. The modulation speed is mainly dependent on the Q factor of an MRR. Device 2 with the designed $K$ of 0.15 has a higher modulation frequency.

$3 \mathrm{~dB}$ cut-off frequencies are decided by both the Q-factors of the MRRs and $R C$ time constants. Because the evaluated $3 \mathrm{~dB}$ cut-off frequencies of Devices 1-2 and 2 dominated by $R C$ time constants $\left(f_{R C}\right)$ are 38 and $35 \mathrm{GHz}$, respectively, the modulation speeds of the devices are mainly limited by the Q factors of the MRRs. As mentioned before, the Q-factors of Devices 1-2 and 2 evaluated from the measured $F_{\text {pe }}$ are approximately 9260 and 8630, respectively. The cut-off frequencies dominated by the Q-factors $\left(f_{\mathrm{Q}}\right)$ are calculated to be 21.0 and $22.4 \mathrm{GHz}$. With the consideration of $f_{R C}$ of Devices 1-2 and 2, the total cut-off frequencies are evaluated to be 13.5 and $13.6 \mathrm{GHz}$, respectively. These values are consistent with the measured 3 -dB cut-off frequencies in Fig. 10.

The experimental results show that the modulation speed of $32 \mathrm{GHz}$ of the proposed MRR-MZM is comparable to typical modulation speeds of 10 to $50 \mathrm{Gbps}$ of a normal MZM. On the other hand, the lengths of the phase shifter are successfully decreased to approximately $1 / 10$ to $1 / 20$ of that of a normal Si MZM.

Si MRRs are very sensitive to changes in temperature and a temperature control system for an MRR is required for practical use. Even with this demerit of the Si MRR, we believe that they are promising because of their compactness and excellent functionality. The MRRs with smart mechanisms of automatic temperature control have been proposed so far. ${ }^{41,42)}$ This is one of the good options to solve this disadvantage.

\section{Conclusions}

We discussed single Si MRR-MZM with a lateral P-N 
junction. Modulation characteristics of two types of MRRMZM with different device parameters, such as the coupling efficiency $K$ and round-trip length of an MRR were investigated. The Si MRR-MZMs were fabricated using a CMOS-compatible process and their high-speed modulation characteristics were compared. A half-wave voltage of $3.4 \mathrm{~V}$ was demonstrated in an MRR-MZM with $K=0.12$ and a round-trip length of $128 \mu \mathrm{m}$, and $V_{\pi} L$ was decreased to $0.036 \mathrm{~V} \mathrm{~cm}$. The $3 \mathrm{~dB}$ bandwidth of an MRR-MZM with $K=0.21$ and a round-trip length of $180 \mu \mathrm{m}$ was measured to be approximately $16 \mathrm{GHz}$, and an NRZ modulation up to $32 \mathrm{Gbps}$ was successfully demonstrated for an operation voltage of $4.0 \mathrm{~V}$.

\section{Acknowledgments}

The authors express sincere thanks to Professor T. Baba and Mr. Y. Hinakura of Yokohama National University for their kind support in the measurements of dynamic modulation characteristics. This work was partly supported by a Grant-inAid for Scientific Research B (No. 15H03577) from the Ministry of Education, Culture, Sports, Science and Technology and by Fujikura Foundation.

1) A. H. Gnauck and P. J. Winzer, J. Lightwave Technol. 23, 115 (2005).

2) T. Kawanishi, S. Sakamoto, and M. Izutsu, IEEE J. Sel. Top. Quantum Electron. 13, 79 (2007).

3) D. Marris-Morini, L. Vivien, J. M. Fédéli, E. Cassan, P. Lyan, and S. Laval, Opt. Express 16, 334 (2008).

4) Z.-Y. Li, D.-X. Xu, W. Ross McKinnon, S. Janz, J. H. Schmid, P. Cheben, and J.-Z. Yu, Opt. Express 17, 15947 (2009).

5) P. Dong, L. Chen, and Y.-K. Chen, Opt. Express 20, 6163 (2012).

6) D. J. Thomson, F. Y. Gardes, J. Fedeli, S. Zlatanovic, Y. Hu, B. Ping, P. Kuo, E. Myslivets, N. Alic, S. Radic, G. Z. Mashanovich, and G. T. Reed, IEEE Photonics Technol. Lett. 24, 234 (2012).

7) H. Xu, X. Xiao, X. Li, Y. Hu, Z. Li, T. Chu, Y. Yu, and J. Yu, Opt. Express 20, 15093 (2012)

8) X. Xiao, H. Xu, X. Li, Z. Li, T. Chu, Y. Yu, and J. Yu, Opt. Express 21, 4116 (2013).

9) X. Tu, T.-Y. Liow, J. Song, X. Luo, Q. Fang, M. Yu, and G.-Q. Lo, Opt. Express 21, 12776 (2013).

10) K. Ogawa, K. Goi, H. Kusaka, K. Oda, T.-Y. Liow, X. Tu, G.-Q. Lo, and D.-L. Kwong, Optical Fiber Communication Conf. Expo. (OFC), 2012, JTh2A.20.

11) P. Dong, L. Chen, C. Xie, L. L. Buhl, and Y.-K. Chen, Opt. Express 20, 21181 (2012)

12) B. Milivojevic, C. Raabe, A. Shastri, M. Webster, P. Metz, S. Sunder, B. Chattin, S. Wiese, B. Dama, and K. Shastri, Optical Fiber Communication Conf. Expo. (OFC), 2013, OTh1D.1.

13) P. Dong, X. Liu, S. Chandrasekhar, L. L. Buhl, R. Aroca, Y. Baeyens, and Y.-K. Chen, 39th European Conf. Exhib. Optical Communication (ECOC), 2013, We.2.B.1.
14) K. Goi, A. Oka, H. Kusaka, Y. Terada, K. Ogawa, T.-Y. Liow, X. Tu, G.-Q. Lo, and D.-L. Kwong, Opt. Express 22, 10703 (2014).

15) D. Korn, R. Palmer, H. Yu, P. C. Schindler, L. Alloatti, M. Baier, R. Schmogrow, W. Bogaerts, S. K. Selvaraja, G. Lepage, M. Pantouvaki, J. M. D. Wouters, P. Verheyen, J. Van Campenhout, B. Chen, R. Baets, P. Absil, R. Dinu, C. Koos, W. Freude, and J. Leuthold, Opt. Express 21, 13219 (2013).

16) P. Dong, X. Liu, C. Sethumadhavan, L. L. Buhl, R. Aroca, Y. Baeyens, and Y.-K. Chen, Optical Fiber Communication Conf. (OFC), 2013, PDP5C.6.

17) J. Ding, S. Shao, L. Zhang, X. Fu, and L. Yang, Opt. Lett. 42, 1636 (2017).

18) H. C. Nguyen, S. Hashimoto, M. Shinkawa, and T. Baba, Opt. Express 20, 22465 (2012).

19) H. Ito, Y. Terada, N. Ishikura, and T. Baba, Opt. Express 23, 21629 (2015).

20) S. Matsuo, Y. Ohiso, and T. Segawa, IEICE Trans. Electron. E88-C, 295 (2005).

21) Y. Kokubun, IEICE Trans. Electron. E90-C, 1037 (2007).

22) F. Xia, M. Rooks, L. Sekaric, and Y. Vlasov, Opt. Express 15, 11934 (2007).

23) J. C. C. Mak, W. D. Sacher, T. Xue, J. C. Mikkelsen, Z. Yong, and J. K. S. Poon, IEEE J. Quantum Electron. 51, 0600411 (2015).

24) Y. Liu, R. Ding, Y. Ma, Y. Yang, Z. Xuan, Q. Li, A. E.-J. Lim, G.-Q. Lo, K. Bergman, T. Baehr-Jones, and M. Hochberg, Opt. Express 22, 16431 (2014).

25) R. Yang, L. Zhou, H. Zhu, and J. Chen, Opt. Express 23, 28993 (2015).

26) W. Bogaerts, P. De Heyn, T. Van Vaerenbergh, K. DeVos, S. K. Selvaraja, T. Claes, P. Dumon, P. Bienstman, D. Van Thourhout, and R. Baets, Laser Photonics Rev. 6, 47 (2012).

27) K. Miura, Y. Shoji, and T. Mizumoto, Jpn. J. Appl. Phys. 55, 068001 (2016).

28) J. E. Heebner, N. N. Lepeshkin, A. Schweinsberg, G. W. Wicks, R. W. Boyd, R. Grover, and P.-T. Ho, Opt. Lett. 29, 769 (2004).

29) T. A. Ibrahim, W. Cao, Y. Kim, J. Li, J. Goldhar, P.-T. Ho, and C. H. Lee, J. Lightwave Technol. 21, 2997 (2003).

30) S. Akiyama, T. Kurahashi, T. Baba, N. Hatori, T. Usuki, and T. Yamamoto, Appl. Phys. Express 3, 072202 (2010).

31) X. Li, X. Feng, K. Cui, F. Liu, and Y. Huang, Opt. Express 22, 10550 (2014).

32) H. Homma, R. Gautam, T. Arakawa, and Y. Kokubun, 20th Microoptics Conf. (MOC '15), 2015, G2.

33) L. Lu, L. Zhou, Z. Li, D. Li, S. Zhao, X. Li, and J. Chen, IEEE Photonics Technol. Lett. 27, 2457 (2015).

34) R. Li, A. Samani, E. El-Fiky, D. Patel, Q. Zhong, and D. V. Plant, Conf. Lasers and Electro-Optics, 2016, STu4G.4.

35) R. Li, D. Patel, E. El-Fiky, A. Samani, Z. Xing, L. Xu, M. Morsy-Osman, and D. V. Plant, Opt. Express 25, 13222 (2017).

36) W. Green, R. Lee, G. DeRose, A. Scherer, and A. Yariv, Opt. Express 13, 1651 (2005).

37) R. Gautam, H. Kaneshige, H. Yamada, R. Katouf, T. Arakawa, and Y. Kokubun, Jpn. J. Appl. Phys. 53, 022201 (2014).

38) H. Kaneshige, Y. Ueyama, H. Yamada, H. Yajima, T. Arakawa, and Y. Kokubun, Jpn. J. Appl. Phys. 51, 02BG01 (2012).

39) H. Kaneshige, R. Gautam, Y. Ueyama, R. Katouf, T. Arakawa, and Y. Kokubun, Opt. Express 21, 16888 (2013).

40) Y. Yabushita, H. Takazawa, Y. Kokubun, and T. Arakawa, 22nd Microoptics Conf., 2017, A-4.

41) E. Timurdogan, C. M. Sorace-Agaskar, J. Sun, E. S. Hosseini, A. Biberman, and M. R. Watts, Nat. Commun. 5, 4008 (2014).

42) K. Padmaraju, D. F. Logan, T. Shiraishi, J. J. Ackert, A. P. Knights, and K. Bergman, J. Lightwave Technol. 32, 505 (2014). 